\title{
HUBBLE SPACE TELESCOPE IMAGING OF $\eta$ CARINAE
}

\author{
J. JEFF HESTER \\ Infrared Processing and Analysis Center, Mail Stop 100-22, California Institute of Technology, Pasadena, California 91125
}

ROBERT M. LIGHT

UCO/Lick Observatories, Board of Studies in Astronomy and Astrophysics, University of California, Santa Cruz, California 95064

JAMES A. WESTPHAL

Division of Geological and Planetary Sciences, Mail Stop 170-25, California Institute of Technology, Pasadena, California 91125

Douglas G. Currie

Department of Physics and Astronomy, University of Maryland, College Park, Maryland 20742

EDWARD J. Groth

Physics Department, Princeton University, Princeton, New Jersey 08544

Jon A. Holtzman

Lowell Observatory, Flagstaff, Arizona 86001

TOD R. LAUER AND EARL J. O'NeIL, JR.

Kitt Peak National Observatory, National Optical Astronomy Observatories, ${ }^{1}$ Tucson, Arizona 85726 Received 17 April 1991

\begin{abstract}
We present new high spatial resolution observations of the material around $\eta$ Carinae obtained with the Hubble Space Telescope Wide Field/Planetary Camera. The star $\eta$ Carinae is one of the most massive and luminous stars in our Galaxy, and has been episodically expelling significant quantities of gas over the last few centuries. The morphology of the bright central nebulosity (the homunculus) indicates that it is a thin shell with very well defined edges, and is clumpy on $0.2\left(\sim 10^{16} \mathrm{~cm}\right)$ scales. An extension to the northeast of the star \{NN/NS using Walborn's [ApJL, 204, L17 (1976)] nomenclature $\}$ appears to be a stellar jet and its associated bow shock. The bow shock is notable for an intriguing series of parallel linear features across its face. The $\mathrm{S}$ ridge and the W arc appear to be part of a "cap" of emission located to the SW and behind the star. Together, the NE jet and the SW cap suggest that the symmetry axis for the system runs NE-SW rather than SE-NW, as previously supposed. Overall, the data indicate that the material around the star may represent an oblate shell with polar blowouts, rather than a bipolar flow.
\end{abstract}

\section{INTRODUCTION}

At a distance of $\sim 2.8 \mathrm{kpc}$ (Humphreys \& Davidson 1984), $\eta$ Carinae is one of the most massive $\left(\gtrsim 100 \mathscr{M}_{\odot}\right.$; Davidson et al. 1986) and luminous $\left(10^{6.6} L_{\odot}\right.$; Westphal \& Neugebauer 1969) stars in the Galaxy. It has been highly variable over at least the last two centuries, and during an outburst in 1843 reached a visual magnitude of -1 , making it the second brightest star in the sky. Although since that time its visual brightness has fallen by a factor of 1000 or so, at a wavelength of $20 \mu \mathrm{m}$ it is still the brightest object in the sky outside of our solar system. The opportunity to study a supermassive star at close range during a period when it is highly unstable makes $\eta$ Carinae a unique and important object.

Surrounding $\eta$ Car is a small nebula consisting of a bright elongated $\left(12^{\prime \prime} \times 17^{\prime \prime}=4.9 \times 10^{17} \mathrm{~cm} \times 6.9 \times 10^{17} \mathrm{~cm}\right)$ structure called the "homunculus" (Gaviola 1950). Both the continuum and broad $\mathrm{H} \alpha$ emission from the homuncu-

${ }^{1}$ NOAO is operated by the Association of Universities For Research in Astronomy, Inc., under cooperative agreement with the National Science Foundation. lus are highly polarized (Meaburn et al. 1987; hereafter referred to as MWW) and the optical extent of the homunculus is coincident with its extent in the thermal infrared (Aitken \& Jones 1975), showing that it is dusty and is seen in the visual primarily in reflected light from the possibly extended photosphere of the central object. A fainter outer shell which shows strong [N II] emission (Walborn 1976; Davidson et al. 1982) and soft x-ray emission (Chlebowski et al. 1984) is thought to be shock ionized CNO-processed ejecta. Proper motions of the homunculus (Gaviola 1950) and the outer shell material (Walborn \& Blanco 1988) generally indicate that this material was ejected during the 1800's. High resolution visible (Burgarella \& Paresce 1991) and infrared (Hackwell et al. 1986; Russel et al. 1987) images show much the same structure, and indicate that the emission around $\eta$ Car is clumpy to the limits imposed by ground based seeing.

\section{OBSERVATIONS AND REDUCTIONS}

The image shown in Fig. 1 [Plate 25] is a composite of seven exposures obtained with the Hubble Space Telescope Wide Field/Planetary Camera (WF/PC) in its Wide Field $(0.1 \mathrm{arcsec} / \mathrm{pixel})$ mode. The instrument is described by Westphal et al. (1982). The star was placed about 10" from 
the corner of WF2 (detector 2 of 4 in the WF), such that the homunculus was contained on WF2 but portions of the outer nebulosity fell on the other chips. Six of the exposures were taken through a narrow band ( $\sim 20 \AA$ FWHM) filter in the light of $[\mathrm{N} \mathrm{II}] \lambda 6584(2 \times 20 \mathrm{~s}, 2 \times 80 \mathrm{~s}$, and $2 \times 400 \mathrm{~s})$. The "Christmas tree" structure and vertical line extending to the north of the star are artifacts of the extreme saturation of the CCD in the center of the object. Diffraction spikes oriented at $45^{\circ}$ and artifacts due to internal reflections are also present at a low level.

The data were flattened using broadband $V$ flats (F658N flats were unavailable). This adequately removed most pixel-to-pixel response variations, but the data did not match up well across CCD boundaries, leading to the cross-shaped artifact to the left and below the star. Image pairs were used to flag and remove cosmic ray events. The six exposures were coadded with weighting appropriate to maximize the signal to noise in the resultant image. The inner 2 " of the image, which was saturated in the shortest $\mathrm{F} 658 \mathrm{~N}$ exposure, was filled in with data from a $0.11 \mathrm{~s}$ deep red exposure which had only three saturated pixels in its core. The images from the four individual chips were rotated and joined to form a mosaic of the region around $\eta$ Car.

The difficulties of the Hubble Space Telescope have been well reported, and discussions of the impact on the scientific objectives that can be achieved with the telescope appear in the literature (e.g., Burrows et al. 1991; Holtzman et al. 1991). To partially correct for the results of spherical aberration the image in Fig. 1 has been deconvolved using the algorithm described by Lucy (1974). The point spread function (PSF) used for deconvolution was appropriate for WF2, leading to noticeable residuals around features in the other three CCDs. Due to the brightness of the star and uncertainties in the PSF, residual effects of the spherical aberration of the HST primary are also pronounced in the center of the object. While the gross structure near the star appears to be consistent with ground-based images (e.g., the bright knot to the NW of the star and the slight extension to the WSW of the homunculus), much of the structure apparent in the inner 2" of Fig. 1 is questionable. In particular it appears that some of the radially oriented structure to the SW of the star is related to structure visible in the inner parts of the PSF.

\section{THE MATERIAL AROUND $\eta$ CARINAE}

\subsection{The Homunculus}

With the unfortunate need for this caveat aside, the WF/PC images reveal much about the structure of the material surrounding $\eta \mathrm{Car}$. The homunculus is seen here to be a well-defined object with a very sharp boundary. There is some indication of limb brightening, especially along the NW and SE edges. The homunculus is resolved into a complex of knots with a characteristic scale of about $0.2-0.3$ arcsec $\left(\sim 10^{16} \mathrm{~cm}\right)$. It is not clear whether the instability responsible for the clumps occurred early in a single episode of mass ejection from the star or whether it is due to the continuing interaction of the stellar wind with circumstellar material. The apparent "beads-on-a-string" organization of the clumps gives the visual impression that magnetic fields may be important in the evolution of the homunculus. Overall, the morphology together with the fact that material at the edge of the homunculus is reflecting light from the central source indicates that the homunculus is a thin shell rath- er than a filled volume. Motion of optically thick clumps in the shell across the line of sight to the star may account in part for the star's continued variability.

The homunculus is pinched or breached along the NE-SW direction, although it does not cleanly separate into two lobes. This structure is also apparent as "horns" visible in high resolution ground-based infrared images (Hackwell et al. 1986). To the NE of the star there are three radial filaments (two of which seem to form a loop) which bisect the indentation in the homunculus. (As mentioned above, we question the reality of much of the radial structure SW of $\eta$ Car.) The morphology of the homunculus may be consistent either with a bipolar structure oriented NW-SE or with a flattened shell.

In the infrared the central source in $\eta$ Car is resolved as a bright hot source coincident with the star and a second cooler source 2" to the NE (Hyland et al. 1979; Hackwell et al. 1986). This source has been interpreted as a dense ring or disk that is providing confinement and collimation for a NW-SE bipolar flow (Warren-Smith et al. 1979; MWW), although a confining disk would not be expected to appear as a one-sided source. If the homunculus is a bipolar structure with its axis of symmetry running NW-SE, then its appearance in the HST images (in particular features such as the edge that comes in from the SE to a point an arcsecond or so to the $\mathrm{N}$ of the star) indicates that it is more like two bubbles joined at the center than a simple conical outflow. This would indicate that the pressure in the interior of the homunculus supplied by the shocked stellar wind has been of at least comparable importance to the momentum of the shell material or the momentum of the wind in determining its dynamical evolution.

\subsection{The Northern Jet}

The structure of the feature to the NE of the homunculus ["NN-NS" using standard nomenclature from Walborn (1976) ] is among the most fascinating aspects of the nebulosity around $\eta$ Car revealed by the WF/PC images. The shape of the envelope of the feature, its sharp brightened edges, and its more diffuse filled interior give it the overall appearance of a limb brightened bow shock. There are two parallel linear features, suggestive of a tubelike structure, extending from the apex of the bow shock exactly back toward the star. This structure is reminiscent in some respects of the Crab Nebula "jet" (Gull \& Fesen 1982). Ground-based spectroscopy (MWW) and proper motion data (Walborn \& Blanco 1988 ) indicate that the apex of this object is moving at a velocity of about $1500 \mathrm{~km} \mathrm{~s}^{-1}$ at an angle of $\sim 25^{\circ}$ out of the plane of the sky. The velocity data appear to unambiguously tie this feature to energetic mass loss from $\eta$ Car, and rule out possibilities such as a "trail" left by the star. The most likely explanation for this feature is that it is a jet of material from $\eta$ Car which is driving a shock into the medium surrounding the star. The tubelike structure could be due to shocks at the walls of the jet. The small ring of emission where the jet intersects the bow shock may be the Mach disk of the jet.

To the SE of the jet there are seven parallel lines of emission oriented perpendicular to the local direction toward $\eta$ Car. This "ladder" structure is visible in the raw data, and is unlike any artifact that we have seen in WF/PC images. We believe that it is real. The nature of the ladder is a puzzle. The regular spacing of the "rungs" $(0$ " $65 \pm 0$ ".05, $1 \sigma)$ suggests a mechanism with a well-defined wavelength. The orientation 
implies a relationship to the outflow from the star. We speculate that if the north extension to $\eta$ Car is in fact a jet and its associated bow shock, then these lines might be "ripples" in the backflow of cooling material along the walls of the bow shock.

\subsection{The South Ridge}

The outer ridge of emission to the SW of $\eta$ Car (the "south ridge;" Walborn 1976) consists of knots and filaments with sizes down to the resolution limit of the image. This morphology is reminiscent of Cas $\mathrm{A}$, and seems consistent with clumpy ejecta hitting slower moving material (Davidson et al. 1986). There is a slight bulge in the ridge to the SW of the star. The region between the ridge and the homunculus is filled in with more diffuse emission. The previously reported "jet" to the SSE of $\eta$ Car (MWW) is present in the HST image as a very sharp feature extending to about 1.5 times the radius of the outer shell. This feature appears to be significantly fainter with respect to the rest of the nebula than it was at the earlier epoch.

We suggest that the south ridge, the "outer shell" material to the SE and NW of the homunculus, and the diffuse emission between the ridge and the homunculus form a "cap" of emission located to the SW and behind the star. The south ridge appears as a ridge because of limb brightening. The cap is roughly rotationally symmetric about an axis close to that of the northern jet. Published data on the velocity field of the outer shell (Walborn et al. 1978; MWW) seem consistent with this picture.

\section{NEW MODEL FOR THE NEBULA}

While the bipolar model seems to work fairly well for the homunculus, it does not explain the structure of the outer portions of the nebula. For example, it is unexpected that the jet and bow shock structure to the NE should lie in very nearly the same plane as the dense collimating disk, as required by this model. In addition, the axis of symmetry of the outer nebula, as defined by the jet and the cap to the SW, appears to be oriented NE-SW, at right angles to the proposed axis of the bipolar flow. As an alternative we propose that the homunculus may be an oblate shell of material with its axis aligned NE-SW and with polar "blowouts" associated with the jet to the NE and the cap to the SW. In this picture the IR source 2" NE of $\eta$ Car could be associated with the inner parts of the polar outflow responsible for the jet. Figure 2 shows a sketch of this proposed structure.

In this model the polar blowouts must be highly asymmetrical. Such is the case for NGC 6888, a large fragmented oblate shell around a Wolf-Rayet star. This shell shows a significantly enhanced N abundance (Parker 1978), and so must be composed largely of processed stellar ejecta which has been expanding under the influence of its own momentum and pressure from the stellar wind. At one pole of the NGC 6888 shell there is a well-defined hole surrounded by "splatter" providing clear evidence of an actual blowout. The opposite side of the shell is not well defined, and data suggest that it has been disrupted (Mitra et al. 1990; Dufour 1989). We suggest that $\eta$ Car may evolve into an object very similar to NGC 6888 .

\subsection{The Velocity Structure}

Among the most compelling data used to support a bipolar model for $\eta$ Car are the observed radial velocities in the

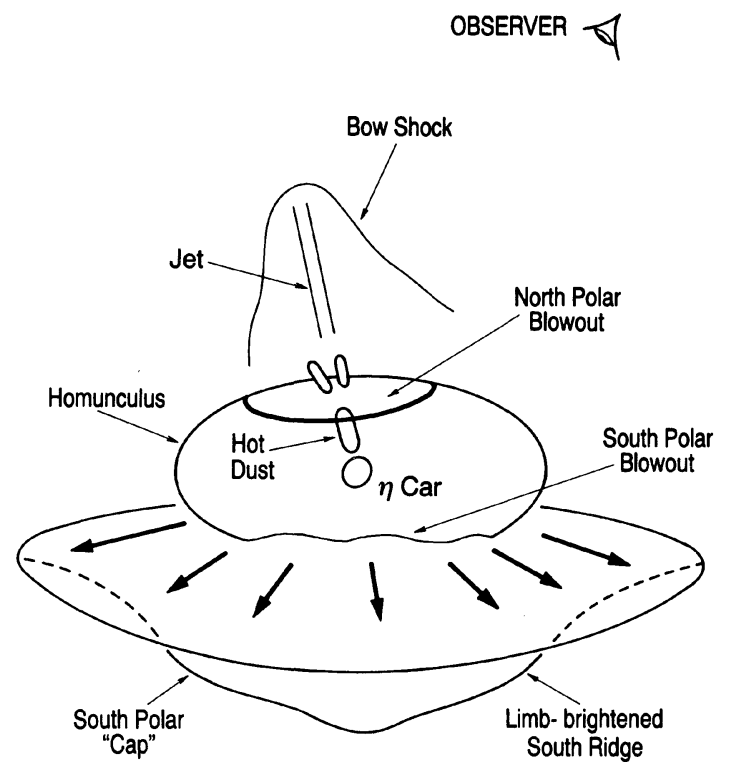

FIG. 2. Sketch showing the proposed shell model for the nebula around $\eta$ Carinae. The homunculus is viewed as an oblate shell rather than a bipolar flow. The northern jet and bow shock, the southern cap, and the homunculus all have approximately the same axis of rotational symmetry. The northern jet and the southern cap are associated with asymmetrical polar blowouts from the shell.

homunculus (MWW). Directly toward the star itself there is a strong broad $\mathrm{H} \alpha$ component at the rest velocity of the system. Off the star this component is redshifted, reaching $+1200 \mathrm{~km} \mathrm{~s}^{-1}$ to the north and $+200 \mathrm{~km} \mathrm{~s}^{-1}$ to the south. We constructed an oblate model of the homunculus to determine if it could duplicate these observations. The shell was modeled as an ellipsoid with an axial ratio of $2: 1$. The polar blowouts were modeled by truncating the shell at $\pm 30^{\circ}$ from the equator. The shell was tipped by $30^{\circ}$ with respect to the plane of the sky to roughly match the orientation of the northern feature and was rotated to match the orientation of $\eta \mathrm{Car}$ in the sky. The shell was assumed to be expanding homologously, and as appropriate for a reflection nebula the velocity observed at a given location was set equal to the expansion velocity at that point plus the line of sight velocity.

Contours of the resulting velocity structure are shown in Fig. 3. The model exhibits a pronounced N-S asymmetry in the same sense as the observations. This asymmetry is primarily due to the fact that we see through to the opposite side of the shell through the northeastern polar hole. A secondary contribution comes from the fact that we are seeing the shell more nearly face on to the SW of the star (resulting in observed velocities in the reflection nebula near 0 ) and more edge on to the NE of the star (resulting in observed velocities in the reflection nebula comparable to the expansion velocity of the shell). An expansion velocity of $700 \mathrm{~km} \mathrm{~s}^{-1}$ at the equator of the shell provides a very good match to the observed $\mathrm{N}-\mathrm{S}$ velocity asymmetry. 


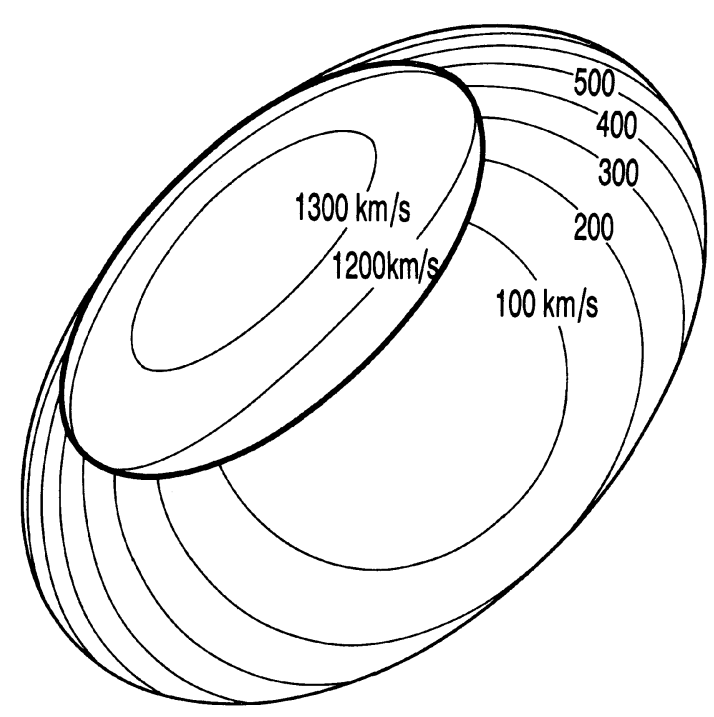

FIG. 3. Velocity field contours for a model describing the homunculus as an optically thick oblate shell with polar blowouts. The axial ratio of the shell is $2: 1$. The shell is complete to $\pm 30^{\circ}$ of the equator, and has been tilted $30^{\circ}$ out of the plane of the sky. The shell is expanding homologously with a velocity of 700 $\mathrm{km} \mathrm{s}^{-1}$ at the equator. The model accounts for highly redshifted emission previously reported to the north of $\eta$ Car as the far side of the shell viewed through the north polar blowout.

In Fig. 1 there is an inverted U-shaped portion of the homunculus immediately to the north of $\eta$ Car which is somewhat fainter than the rest of the shell. This may be where we are seeing through the polar hole to the far side of the shell. On the other hand, the limb brightened southeastern edge of the homunculus can be followed around to the east and in to a point just north of the star, indicating that all of the emission in the SE "lobe" is on the front face of the shell. The jet is also tipped slightly to the north of the proposed axis of the homunculus. Together, these data suggest that the NE blowout may be slightly displaced from the axis of the shell. Seeing through to the far side of the shell to the north but not to the east of $\eta$ Car may account for the differences in line profiles between positions " $\mathrm{X}$ " and " $\mathrm{Y}$ " from MWW.

The velocity structure of the homunculus provides a good way of discriminating between the bipolar and oblate shell models for $\eta$ Car, since the oblate shell model predicts a strong NE/SW asymmetry in the velocity structure which is not predicted by the bipolar outflow model. The velocity data do in fact show an E-W asymmetry in the homunculus in the sense predicted, but the data as published are not adequate to unambiguously distinguish between the two cases.

\subsection{The Morphology of the Homunculus}

The pinched appearance of the homunculus (most notable to the NE and less reliable to the SW) is the most difficult aspect of the data to understand within the context of an oblate shell model. Both the observed morphology and the velocity data require a somewhat complex geometry for the blowout regions. As discussed above for the bipolar model, the observed structure may require that the pressure of the shocked wind be important in the evolution of the shell. It seems very likely that the structure of the homunculus reflects the symmetry of the wind from the star, which could be influenced by the rotation and/or magnetic field structure of the star. An oblate shell might also arise from mass loss from a close binary (Gallagher 1989). The existence of a ring around SN1987A (Jakobsen 1991) shows that mass loss from massive stars can have a toroidal symmetry.

While not all aspects of $\eta$ Car are easily accounted for by an oblate shell model, this model seems more successful than the bipolar outflow model in a number of respects. Distinguishing between these two models should provide a focus for future observational work on this fascinating object.

This research was supported by NASA through various contracts and grants to the WF/PC science team. J.J.H. acknowledges NASA and Caltech support to IPAC.
Aitken, D. K., and Jones, B. 1975, MNRAS, 172, 141

Burgarella, D., and Paresce, F. 1991, A\&A, 241, 595

Burrows, C. J., Holtzman, J.A., Faber, S. M., Bely, P. Y., Hasan, H., Lynds, C. R., and Schroeder, D. 1991, ApJL, 369, L21

Chlebowski, T., Seward, F. D., Swank, J., and Szymkowiak, A. 1984, ApJ, 281,665

Davidson, K., Dufour, R. J., Walborn, N. R., and Gull, T. R. 1986, ApJ, 305,867

Davidson, K., Walborn, N. R., and Gull, T. R. 1982, ApJL, 254, L47

Dufour, R. J. 1989, RMxA, 18, 87

Gallagher, J. S. 1989, in Physics of Luminous Blue Variables, edited by K. Davidson, A. F. J. Moffat, and H. J. G. L. M. Lamers (Kluwer, Dordrecht).

Gaviola, E. 1950, ApJ, 111, 408

Gull, T. R., and Fesen, R. A. 1982, ApJL, 260, L75

Hackwell, J. A., Gehrz, R. D., and Grasdalen, G. L. 1986, ApJ, 311, 380

Holtzman, J. A., et al. 1991, ApJL, 369, L35

Humphreys, R. M., and Davidson, K. 1984, Sci, 223, 243

\section{REFERENCES}

Hyland, A. R., Robinson, G., Mitchell, R. M., Thomas, J. A., and Becklin, E. E. 1979, ApJ, 233, 145

Jakobsen, P. 1991, ApJL, 369, L63

Lucy, L. B. 1974, AJ, 79, 745

Meaburn, J., Wolstencroft, R. D., and Walsh, J. R. 1987, A\&A, 181, 333

Mitra, P. M., Dufour, R. J., Parker, R. A. R., and Hester, J. J. 1990, BAAS, 22, 1251

Parker, R. A. R. 1978, ApJ, 224, 873

Russel, R. W., Lynch, D. K., Hackwell, J. A., Rudy, R. J., Rossano, G. S., and Castelaz, M. W. 1987, ApJ, 321, 937

Walborn, N. R. 1976, ApJL, 204, L17

Walborn, N. R., and Blanco, B. M. 1988, PASP, 100, 797

Walborn, N. R., Blanco, B. M., and Thackeray, A. D. 1978, ApJ, 219, 498 Warren-Smith, R. F., Scarott, S. M., Murdin, P., and Bingham, R. G. 1979, MNRAS, 187, 761

Westphal, J. A., et al. 1983, The Space Telescope Observatory, IAU 18th General Assembly, Patras, Greece, August, 1982 (NASA CP-2244) Westphal, J. A., and Neugebauer, G. 1969 ApJL, 156, L45 


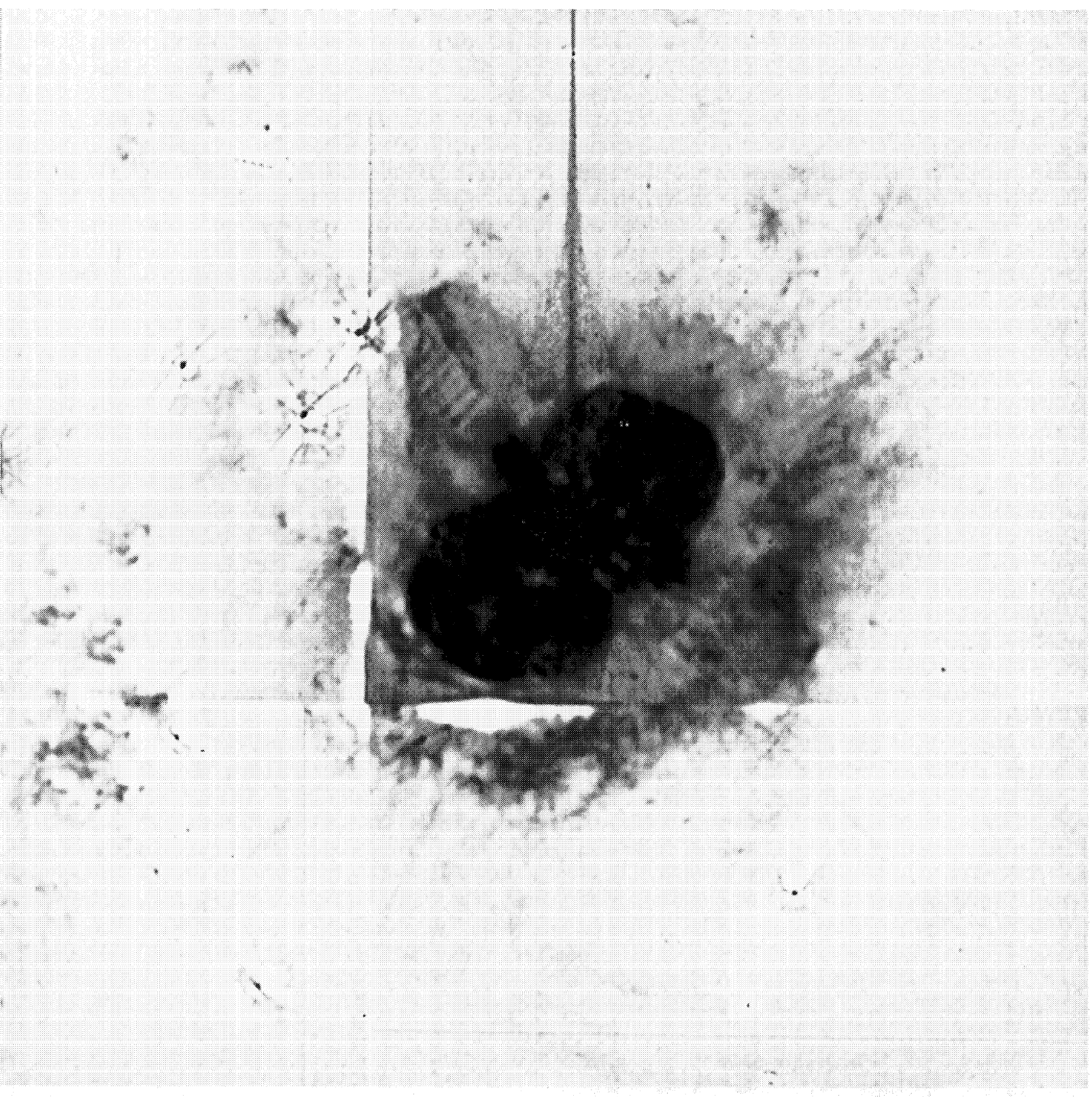

FIG. 1. Hubble Space Telescope Wide Field/Planetary Camera image of the nebulosity around the star $\eta$ Carinae. The orientation is roughly north to the top and east to the left. The field shown in $51^{\prime \prime}$ on a side, or $2 \times 10^{18} \mathrm{~cm}$ at a distance of $2.8 \mathrm{kpc}$. The data have been processed to partially correct for the effects of the spherical aberration in the telescope but caution is required in interpreting features near the center of the nebulosity. The data are displayed with a logarithmic stretch.

Hester et al. (see page 654) 